\title{
Aider les aidants : quel espace pour l'innovation sociale dans le soutien aux proches aidants en région? ${ }^{1}$
}

\author{
Marco Alberio ${ }^{\mathbf{a}}$
}

RÉSUMÉ. Dans cet article, nous examinons les initiatives et actions locales visant à réduire les inégalités découlant du rôle de proche aidant tant à l'échelle individuelle, collective (de groupes spécifiques, tels que les femmes) que territoriale. Nous présenterons les résultats d'une étude qualitative réalisée au Québec en 2015 auprès de proches aidants d'ainés qui occupent un poste à temps plein dans le marché du travail, ainsi qu'auprès des professionnels leur offrant des services. Plus précisément, nous observerons comment différents acteurs (institutions de la santé, associations, MRC, etc.) essaient de mettre en œuvre et de conserver une offre de services pour les proches aidants, et comment ces services et ces initiatives peuvent affecter la vie quotidienne des aidants en leur permettant, en premier lieu, de s’identifier comme proches aidants et, plus largement, en influençant leurs trajectoires et expériences de conciliation entre travail, famille et soins.

ABSTRACT. In this article, we examine local initiatives and actions aimed at reducing individual, collective (specific groups, such as women) and territorial inequalities resulting from the role of caregiver. Included are the results of a qualitative study carried out in Quebec in 2015 with elderly people caregivers working full-time, as well as with professionals providing them services. More specifically there are observations on how different actors (health institutions, associations, regional county municipalities, etc.) try to implement and maintain services for caregivers, and how these services and initiatives can affect the daily lives of caregivers by allowing them firstly to identify themselves as caregivers and then by influencing their paths and experiences in balancing work, family and care.

\section{Introduction}

Dans cet article, nous allons discuter de la mise en œuvre d'initiatives pour le soutien des proches aidants d'ainés, et de la façon dont ces services peuvent affecter la vie quotidienne des aidants et influencer leurs trajectoires et expériences de conciliation travail-famille-soins dans la région du Bas-Saint-Laurent. Ce faisant, nous prenons en compte les contextes démographique, social et politique (à différents paliers) dans lesquels ces initiatives et services ont vu le jour, ainsi que les conditions pour les maintenir.

Le cas du Québec, eu égard aux offres de services pour proches aidants, est intéressant pour plusieurs raisons. Premièrement, à partir de la fin des années 1990, la province a élaboré d'importantes politiques en matière de conciliation de la vie familiale et professionnelle (Tremblay, 2016). Deuxièmement, le Québec est un vaste territoire faisant face à des défis associés au vieillissement de sa population ainsi qu'à l'accessibilité aux soins et aux services sociaux sur l'ensemble de son territoire (Lavoie et Guberman, 2009). La tradition d'initiatives sociales à l'échelle locale est un autre aspect important au Québec (Alberio et Mbaye, 2015; Moralli, Alberio et Klein, 2017). Cette tradition a un fort impact sur la structuration des initiatives et sur le rôle des différents acteurs impliqués.

Notre étude de cas concerne la région du Bas-SaintLaurent - en particulier la municipalité régionale de comté (MRC) de Rimouski-Neigette et celle plus rurale de La Mitis -, qui compte 199977 habitants sur une superficie de $22234 \mathrm{~km}^{2}$, dont presque le quart (46 860) est à Rimouski (ISQ, 2015). Dans

${ }^{\text {a }}$ Chaire de recherche du Canada en innovation sociale et développement des territoires, Université du Québec à Rimouski 
certaines parties de cette région, la densité de population atteint à peine 22 personnes par $\mathrm{km}^{2}$. Ces caractéristiques spatiales peuvent selon nous affecter les expériences et pratiques des proches aidants, et auraient également des conséquences spécifiques sur la dispensation des services par les intervenants ainsi que sur leur utilisation par les aidants.

Dans le contexte du virage des années 1990-2000 vers les soins ambulatoires au Canada, comme dans d'autres pays, les experts ont constaté une tendance générale à prioriser l'environnement social de la personne âgée comme le meilleur endroit pour demeurer et pour recevoir des soins (Gagnon et collab., 2001; Ducharme, 2006). Cette «stratégie» impose toutefois une lourde responsabilité aux membres de la famille, en particulier aux femmes, et présente plusieurs limites. Les Canadiens bénéficiaires d'aide reçoivent souvent leur appui de sources informelles et de membres de leur famille (Chen et Wilkins, 1998; ISQ, 2015). Il ressort également de ces études que les bénéficiaires d'aide ont moins recours aux services privés ou publics lorsqu'un aidant familial est présent.

Dans cette perspective, au Québec et au Canada, une reconnaissance progressive de la situation des proches aidants est apparue dans les politiques publiques et les programmes gouvernementaux, ainsi qu'à l'échelle communautaire. L'innovation sociale par le biais d'initiatives locales peut en fait devenir décisive dans les secteurs public, privé et communautaire/bénévole. Les initiatives socialement innovantes peuvent devenir des solutions aux effets pervers (changements démographiques, inégalités sociales, exclusion et marginalisation territoriale, etc.) de la transformation socioéconomique ayant affecté les sociétés contemporaines. Elles peuvent enfin répondre au besoin de redéfinition du rôle des trois principaux acteurs de la régulation sociale: l'État, le marché et la société civile - y compris la famille (Klein, Fontan, Harrisson et Lévesque, 2014; Moralli et collab., 2017).

La mobilisation des communautés locales à l'aide de différents outils, tels que l'économie sociale, les associations ou les interventions participatives, fournit un cadre pour la reconstruction des liens sociaux (Courlet et Pecqueur, 2013). Cependant, cela soulève la question des limites de la capacité des communautés à influencer totalement les décisions qui les concernent, ainsi que la gouvernance quotidienne et la durabilité des innovations sociales produites localement.

En ce qui concerne plus spécifiquement le soutien aux proches aidants, comme nous le verrons plus loin, le Québec a connu depuis 2010 une mobilisation importante autour de la question des proches aidants de personnes âgées. Cette approche a été encouragée aux échelles nationale et régionale, notamment avec la création de l'organisme à but non lucratif national L'Appui, qui possède des pôles dans plusieurs régions du Québec et qui est financé par le gouvernement provincial, en association avec la fondation privée Lucie et André Chagnon.

\section{Un survol du phénomène de la proche aidance}

La Commission canadienne des droits de la personne (CCDP) définit les proches aidants comme des individus fournissant des soins informels et non rémunérés à domicile et offrant une assistance régulière à un proche, soit-il un jeune enfant, une personne handicapée, une personne âgée, etc. :

Un proche aidant est une personne qui s'occupe d'un jeune enfant, aide quelqu'un ayant une déficience, intervient lors d'un accident ou d'une maladie grave ou veille à combler les besoins d'une personne en fin de vie, par exemple. (CCDP, 2014, p. 2)

Selon le gouvernement du Canada, un proche aidant est généralement une personne de 45 ans ou plus qui a aidé un ou plusieurs membres de sa famille, voisins, amis ou collègues souffrant d'un problème de santé ou d'une limitation physique à long terme (Sinha, 2013). Les services fournis par les aidants peuvent inclure le transport, l'épicerie, les tâches domestiques ou d'entretien extérieur, les soins personnels, les soins ou traitements médicaux, l'organisation des soins et le soutien émotionnel ou moral (Sinha, 2013). L'Institut de la statistique du Québec (ISQ) a signalé qu'en 2012, parmi les Québécois de 45 ans ou plus, le nombre de personnes prodiguant des soins informels atteignait 843000 , sur une population totale de 8,2 millions d'habitants. Un individu sur quatre de ce groupe d'âge fournissait des soins informels (ISQ, 2013). Les aidants sont principalement - mais pas uniquement des femmes. De plus en plus d'hommes se retrouvent dans cette situation et semblent, au moins en partie, faire face à des défis spécifiques, surtout en ce qui concerne la reconnaissance sociale de ce rôle. 
L'assistance et les soins non rémunérés prodigués à une personne proche ont fait l'objet de plusieurs études dans les domaines de la sociologie, des soins de la santé et des services sociaux au Canada (Cranswick et Dosman, 2008; Duxbury, Higgins et Schroeder, 2009; Fast et collab., 2011; Tremblay et Larivière, 2013). Les analyses sociales qualitatives ont mis l'accent sur les perceptions des aidants de leur propre situation et sur les tâches qu'ils doivent accomplir, tandis que la recherche quantitative a principalement examiné les facteurs de risque et les conditions générales des soins. Des recherches européennes ont également montré que l'emploi des femmes (le fait d'être à l'emploi ou non et le nombre d'heures travaillées) est négativement lié aux soins non rémunérés auprès de personnes âgées (Kotsadam, 2011). Les soins peuvent également devenir un défi pour les employeurs, et ce, surtout dans un contexte qui est au moins en partie caractérisé par une rareté de la main-d'œuvre.

Le contexte économique pourrait renforcer la relation entre soins et vulnérabilité. Pour certaines populations, le fait de prodiguer des soins peut également constituer un moment tournant critique dans une trajectoire personnelle et professionnelle déjà fragile. C'est particulièrement le cas pour les femmes de 45 à 65 ans, notamment celles qui se trouvent sur le marché du travail ou sur la marge de celui-ci, c'est-à-dire qui ont des emplois à temps partiel (sans forcément les choisir), de qualité médiocre ou à statut temporaire (Alberio, 2014). Pour ces raisons, les initiatives visant à améliorer les conditions de vie des aidants sont devenues des interventions stratégiques dans le contexte socioéconomique actuel.

\section{Vers de nouvelles perspectives de l'État-providence et de l'intervention sociale}

Dans la plupart des pays occidentaux, le monopole des « frontières nationales » est aujourd'hui particulièrement faible par rapport au passé. Devant le manque de capacités des États-providence nationaux, une réponse des systèmes locaux de protection sociale semble être une solution pour relever les défis émergents, tels que le déséquilibre démographique croissant, l'inégalité persistante entre les hommes et les femmes dans l'accès au marché du travail, la pauvreté et l'exclusion sociale, les emplois précaires pour les jeunes générations et les problèmes liés au marché du logement (Ranci, 2005).

La plupart de ces problèmes sociaux ont en effet une dimension territoriale claire et un impact local fort. Les dispositifs traditionnels sont souvent insuffisants et impliquent parfois des obstacles administratifs et bureaucratiques, des structures rigides et des systèmes d'intérêt privé; des éléments qui agissent comme obstacles au changement social (Ranci, 2005). En même temps, nous ne devrions pas non plus trop dépendre du «local» ni tenir pour acquis que les politiques locales et territoriales peuvent traiter n'importe quel problème social. Une articulation multiniveau des interventions nationales, régionales et locales est donc nécessaire (Kazepov, 2010).

D’une part, les questions d'inégalités territoriales doivent être abordées; d'autre part, il est essentiel d'aller au-delà d'un simple transfert de fonds et du cadre centralisé ${ }^{2}$ présent dans la plupart des Étatsprovidence, qui pourrait également être reproduit à une plus petite échelle, soit régionale. Les défis en matière d'intervention locale concernent à la fois le contenu des politiques et leur mise en œuvre.

Un premier problème concerne la fragmentation : les initiatives sociales locales manquent souvent d'un cadre d'intervention cohérent et homogène, et risquent d'aggraver les inégalités territoriales, en particulier dans des contextes caractérisés par la fragilité et par la marginalité.

Deuxièmement, l'orientation redistributive des États-providence traditionnels doit être adaptée au contexte de contrainte de ressources. Un nouvel équilibre plus efficace entre les objectifs, les nouveaux risques sociaux et les ressources est nécessaire et, eu égard à ces ressources limitées, la définition des critères d'inclusion et d'exclusion peut être une source de conflit à l'échelle locale également.

Un autre enjeu majeur concerne les nouveaux accords contractuels, qui imposent des dispositifs différents et attribuent de nouvelles compétences. Dans ce contexte, il est nécessaire d'avoir une définition commune des objectifs et procédures des différents acteurs, des formes de contrôle, des procédures de suivi et d'évaluation (Pavolini, 2003), en particulier en ce qui concerne la manière dont les responsabilités sont partagées entre les acteurs publics et la communauté ${ }^{3}$, et entre les différents 
acteurs au sein de la communauté. Cela nécessite la participation de nombreux acteurs à un véritable processus décisionnel et à la définition précise des rôles et des responsabilités.

Pour revenir au cas spécifique du Québec, la relation entre l'État et les autres acteurs est également centrale. Après une première implémentation (formelle) d'organismes communautaires dans les décennies 1960 et 1970, qui a joué un rôle important dans la mise en œuvre de l'État-providence québécois, la province est arrivée à la fin des années 1990 à un moment décisif pour la reconnaissance de l'action communautaire et bénévole.

En 1997, le gouvernement provincial a organisé un vaste processus participatif de réorganisation réunissant la plupart des acteurs socioéconomiques de la province 4 . Cela a donné lieu à un courant d'initiatives, telles que le Chantier de l'économie sociale, les Tables de concertation des ainés structurées aux paliers national, régional et local ainsi que les Municipalités amies des aînés. L'objectif était de passer «d'une période où prédominaient l'expérimentation et les projets pilotes à une autre période où leur diffusion à l'échelle de la société devient possible » (Lévesque et Mendell, 2014, cités dans Alberio et Tremblay, 2014, p. 138).

Ce passage de projets fragmentés à un effort de mise en œuvre concerté n'est cependant pas évident et soulève encore des questions importantes. Pour s'attaquer à la fragmentation des initiatives, il est également nécessaire de développer des interventions qui traitent simultanément différents enjeux de politiques sociales (Ranci, 2005). Au Québec, avec ses régions rurales et éloignées, ces interventions peuvent concerner des défis démographiques et le besoin contextuel d'attirer une nouvelle population/main-d'œuvre; des politiques d'activation de l'emploi et de rétention des travailleurs qualifiés; des politiques de mobilité et de transport; et l'amélioration de l'offre de services de santé et sociaux. La clé est de mettre en œuvre des programmes sociaux concrets et complexes à travers une négociation entre les différents acteurs impliqués.

Comparativement à l'État-providence traditionnel, les initiatives locales devraient offrir des solutions plus innovantes, rapides et flexibles selon les besoins sociaux émergents. Les interventions peuvent également tirer profit de la proximité physique et sociale, mais les acteurs locaux font souvent face à des difficultés majeures. Par exemple, bien que le secteur associatif et communautaire soit traditionnellement bien reconnu par les acteurs publics québécois, des ambiguités subsistent dans ses relations avec l'État, jusqu'aux derniers changements survenus en 2015 :

Dans cette foulée, les enjeux sociaux se sont déplacés : la demande de reconnaissances des nouveaux acteurs étant acquise, la demande de démocratisation et d'autonomie n'est pas résolue pour autant. De ce point de vue, l'autonomisation, la représentativité et le nouveau mandat du Chantier de l'économie sociale pourraient répondre au danger de la fragmentation et du corporatisme. (Lévesque et Mendell, 2014, p. 183)

\section{Méthode}

Notre étude avait deux objectifs principaux. Premièrement, nous voulions analyser les expériences des proches aidants en matière de conciliation travail-famille-soins. Deuxièmement, nous voulions étudier le développement et la mise en œuvre de services et d'initiatives pour les aidants aux paliers national, régional et local. Nous avions donc deux populations cibles : les proches aidants et les professionnels de diverses organisations et institutions qui leur fournissent des services. Cet article ne s'appuie que sur une petite partie du matériau analysé. Cependant, ces éléments empiriques permettent d'établir un lien entre l'offre de services (l'objet de notre article) et les besoins et expériences des aidants.

Les questions de recherche de notre étude sont les suivantes: Comment les aidants qui travaillent gèrent-ils leur situation? Comment perçoivent-ils et vivent-ils leur rôle? Comment parviennent-ils à articuler leurs différentes responsabilités?

Concernant les services et l'aide offerts aux proches aidants, nous avons essayé de comprendre comment ces initiatives ont été créées et structurées, ainsi que leur rôle et leur importance pour les aidants. Nous voulions également identifier leurs limites, y compris à l'égard des contextes local et national, afin de déterminer les moyens possibles pour améliorer cette offre de services et de promouvoir une meilleure conciliation travailfamille-soins. 
Pour la collecte de données, nous avons effectué des entretiens semi-structurés (de 1 h 30 à 2 h chacun en moyenne). Nous les avons structurés autour de thèmes qui ont été introduits dans l'entretien sous forme de questions générales. Si nécessaire, nous avons poursuivi avec des questions plus spécifiques afin de cibler les éléments clés de leurs expériences : conciliation travail-famille-soins pour les proches aidants, et la mise en place et la disposition des services pour les professionnels consultés.

Au total, 20 proches aidants ont été interrogés. Le critère de sélection était d'être en emploi, tout en prodiguant des soins à au moins une personne âgée membre de sa famille en tant que principal aidant pendant au moins $5 \mathrm{~h}$ par semaine.

Pour les professionnels, le critère était de travailler dans une organisation offrant des services ou du soutien aux proches aidants et d'être directement impliqué dans cette offre de services. Nous avons rencontré 12 gestionnaires ou intervenants provenant de 8 organismes différents dans la région du Bas-Saint-Laurent.

\section{Une réponse aux nouveaux besoins sociaux}

Comme nous l'avons mentionné dans notre introduction, de nouvelles initiatives pour les proches aidants émergent à la suite du " virage ambulatoire » des années 1990 au Québec. A partir de ce moment, les services de santé et les services sociaux destinés aux personnes âgées ont été progressivement réorientés vers leur domicile et dans leur famille, le domicile devenant, dans le discours politique et social officiel, le meilleur environnement pour les soins des personnes âgées (Ducharme, 2006). Ce nouveau modèle organisationnel a favorisé une restructuration des soins et des services offerts aux personnes âgées, impliquant plus de responsabilités pour les membres de la famille.

C'est au courant des années 1990 que les premières demandes de services et d'appui ont été adressées auprès des services de santé et des services sociaux au Québec de la part des proches aidants. Les intervenants travaillant avec des personnes âgées ont été les premiers à recevoir ces demandes et à répondre à ces besoins, d'abord de manière informelle, sur les plans individuel et local. Ces intervenants ont commencé à partager leurs expériences, initialement au sein de leur organisme respectif (secteur communautaire ou institutions publiques), ensuite au sein de leur réseau régional plus vaste de soins gériatriques.

Plusieurs groupes de travail et comités pour des projets pilotes ont été créés aux échelles locale et régionale pour rassembler les principaux acteurs œuvrant auprès des personnes âgées. Au sein de ces groupes et comités, un intérêt spécifique pour les proches aidants s'est clairement manifesté :

En fait, la Table des proches aidants des personnes aînées est née de l'élaboration, à l'époque, de projets cliniques. Un des besoins qui ressortaient, c'était le soutien aux proches aidants dans ce projet clinique, avec une attention particulière aux proches aidants d'une personne aînée atteinte de la maladie d'Alzheimer ou d'un autre type de déficit cognitif. C'est un peu de là qu'a émergé cette table. Autour de 2006 ou 2007, je ne pourrais pas dire exactement, elle a eu des vagues; elle a vraiment eu des vagues. (Entretien 03, intervenante, services de santé)

Vers le milieu des années 2000, des initiatives territoriales rassemblant des acteurs publics (hôpitaux, services sociaux, etc.) et communautaires ont vu le jour afin de répondre formellement à ces «nouveaux » besoins et risques sociaux. Cette prise de conscience a également atteint des institutions publiques, dont le Centre de santé et des services sociaux $(\mathrm{CSSS})^{5}$ du Bas-Saint-Laurent :

J'ai l'impression que ça a commencé à partir du moment où le CSSS s'est rendu compte d'une faille dans son système, où les intervenants à domicile se rendaient compte finalement qu'ils travaillaient beaucoup plus en collaboration avec des proches aidants qu'avec des aidés en tant que tels. Que tout le système est en fonction des aidés et que les aidants, eux, quand il y avait des besoins, ils n'étaient pas considérés comme des patients ou comme de la clientèle du CSSS; c'était l'aidé. C'est à partir de l'aidé qu'on déterminait tous les soins qui allaient être apportés ou tous les programmes possibles, alors que la réalité de l'aidant était complètement absente. Oui, elle était complètement négligée. Toi, tu as besoin de travailler à l'extérieur, mais il n'y a aucun lien avec les besoins de ton aidé. S'il a l'Alzheimer et a droit à $15 \mathrm{~h}$ par semaine de 
répit, eh bien, toi, tu continues de travailler. Mais si c'est juste le vieillissement, puis qu'il a droit à $3 \mathrm{~h}$ par semaine, eh bien, toi, tu as quand même besoin de travailler. Donc, ça met un certain « clash » dans l'intervention des intervenants. (Entretien 05, intervenant, services institutionnels)

La préoccupation initiale, développée de manière informelle au palier local, a progressivement atteint le palier institutionnel régional, en particulier la Conférence régionale des élus (CRÉ) ${ }^{6}$, une institution intermédiaire composée d'élus et de divers acteurs de la société civile (Alberio, 2015). La prise de conscience du besoin d'offrir des services spécifiques pour les proches aidants a ensuite atteint l'échelle nationale, grâce aussi à des rapports (entre autres celui de Gagnon et Savoie, 2008) et à des initiatives publiques ayant fait émerger les problèmes éprouvés par les proches aidants :

Les proches aidants qui sont venus témoigner ont fait écho à ces difficultés. Plusieurs ont dit avoir un urgent besoin de soutien psychologique et technique. Une grande proportion d'entre eux a aussi fait état de la difficulté à concilier les responsabilités familiales et professionnelles. Cela les a souvent amenés à faire des choix déchirants, comme réduire leur temps de travail ou laisser tomber carrément leur emploi. Enfin, plusieurs groupes soulignent la dure réalité des proches aidants. (Gagnon et Savoie, 2008, p. 51)

Au palier national, le gouvernement a réagi en introduisant des actions - surtout en santé destinées aux proches aidants et en finançant des programmes spécifiques répondant à leurs besoins, mais souvent dans une perspective de « réparation », plutôt que de prévention. L’Assemblée nationale du Québec a adopté la Loi instituant le fonds de soutien aux proches aidants (Québec, 2009), établissant ainsi un partenariat financier entre le gouvernement du Québec et la Fondation Lucie et André Chagnon. Le fonds a été doté d'un investissement total de 200 millions de dollars canadiens et a permis la création de L'Appui, un organisme de gestion du soutien aux proches aidants.

Au cours des années qui ont suivi, cet organisme à but non lucratif à portée nationale, dont le siège national reçoit, administre et redistribue les fonds reçus du ministère de la Famille et de la Fondation, a soutenu la création d'antennes dans les 17 régions administratives du Québec.

La mobilisation dans la région du Bas-SaintLaurent, pilotée par la CRÉ, a abouti à la création de l'antenne régionale L'Appui Bas-Saint-Laurent. Le financement dorénavant disponible a incité certaines institutions et certains organismes communautaires à soumettre des demandes de subvention et ainsi à créer des initiatives spécifiques pour les proches aidants. Cette approche était novatrice, car, auparavant, la plupart des organismes ne proposaient pas d'interventions ni de services spécifiques aux proches aidants. Les politiques publiques et les organismes concentraient leurs efforts - et le font encore souvent aujourd'hui beaucoup plus sur la personne âgée en tant que telle que sur son proche aidant.

Selon un travailleur social, la disponibilité de fonds a permis un travail d'intervention sociale concret qui répond plus directement aux besoins des aidants :

Je me rendais compte qu'il y avait beaucoup de femmes... C'était des situations qu'elles vivaient soit avec leur père, leur mère, leur conjoint, et elles trouvaient ça lourd. Donc, on s'est dit qu'il fallait faire quelque chose avec elles, ces dames-là. C'est sûr qu'on a mené... Moi, j'ai fait de l'intervention et tout ça, sauf qu'il n'y avait pas d'activité spécifique en tant que telle pour les proches aidants. À un moment donné, on a entendu parler pendant quelques années de L'Appui Bas-SaintLaurent, qui offrait des subventions pour aider spécifiquement les proches aidants. À ce moment, on a demandé un projet et ça a été accepté. (Entretien 02, intervenante, organisme communautaire)

\subsection{Vers un regard critique}

Malgré le développement très positif d'initiatives spécifiques destinées aux proches aidants (groupes de soutien psychosocial et d'entraide, séances d'information, temps libre et services de relève), certains obstacles et limites persistent. Une première question importante concerne «l'identité » ou l'(auto)reconnaissance en tant qu'aidant. C'est un facteur décisif dans les conditions de l'aide et dans l'expérience de conciliation travail-famillesoins. L'identité est liée à la reconnaissance générale 
et à la reconnaissance de soi, deux facteurs étroitement liés. Ce manque de reconnaissance dépend du fait que les soins sont souvent ancrés dans des relations familiales et affectives. Dans cette perspective, le genre peut également jouer un rôle. Pour cette raison, les aidants eux-mêmes, leur famille ainsi que les acteurs publics tiennent pour acquis le soutien offert par les proches. Les aidants (surtout les femmes) voient souvent leur contribution comme naturelle, basée sur l'amour et l'affection.

Ce manque de reconnaissance par soi-même et par les autres peut être encore plus évident quand une personne fournit des soins à un conjoint ou conjointe. La prestation de soins au sein d'un couple a souvent de fortes répercussions sur la relation. La personne aidante alterne son rôle de partenaire amoureux et celui de proche aidant. Le rôle de partenaire amoureux est souvent mis de l'avant, par amour et par affection, et peut ainsi cacher l'identité et la reconnaissance en tant qu'aidant. Par exemple, une femme dans une relation avec un homme plus âgé souffrant d'un handicap physique explique comment les rôles d'aidante et d'amoureuse se confondent souvent :

Parce que c'est l'amour fou, comme vous pouvez voir! J'ai les yeux brillants! Ça fait que c'est ça. C'est sûr que, des fois, j'ai envie de crier haut et fort : «Hé! [...] Je ne suis pas juste en amour! » (Entretien 06, aidante, fin quarantaine, travailleuse)

Les sentiments et la dimension affective en général jouent un important rôle dans les relations de soins entre proches, ce qui explique parfois la réticence des aidants à chercher de l'aide extérieure et à l'accepter et, conséquemment, la difficulté qu'ont les organismes à joindre certains proches aidants. Ces derniers craignent la perte du contrôle de leur vie et de leur milieu de vie :

L'aide que je n'accepte pas, c'est pour le ménage, faire à manger. Ça, c'est à faire, mais moi, je ne l'accepte pas. Je ne suis pas à mon aise là-dedans. Si, un jour, ça vient que je ne peux plus faire ça, je ne l'accepterai pas plus. Je changerai d'ambiance. Je m'en irai en chambre et pension. Même si moi, je suis autonome. Et pendant ce temps-là, lorsque la personne sera avec moi en chambre et pension, moi, je serai libre. (Entretien 05, aidant, fin soixantaine)
La question de l'identité et de la reconnaissance des aidants affecte directement l'offre de services à leur égard. La manière dont les aidants se perçoivent et se reconnaissent eux-mêmes a un impact sur les pratiques et le travail quotidiens des professionnels et intervenants qui offrent ces services :

Je pense qu'on pensait tous que les proches aidants allaient être derrière la porte, en train de pousser pour aller chercher les services, alors que, dans les faits, quand on a commencé à déployer les services, on a eu, dans toutes les régions du Québec, un problème. Le concept de proches aidants, [...] c'est pas un concept qui est automatiquement perçu. [...] C'est problématique quand tu offres des services à des proches aidants, mais que les personnes ne se reconnaissent pas dans ce rôle-là. Ça fait qu'ils ne font pas la connexion entre les services disponibles et leur rôle. (Entretien 01, directrice)

Cet enjeu de la reconnaissance sociale et de soimême devient encore plus important lorsque les intervenants, les gestionnaires d'institutions publiques ou les acteurs communautaires souhaitent développer des initiatives selon une approche ascendante (bottom-up) en intégrant, dès le départ, dans le processus d'élaboration des services, les utilisateurs finaux. Un des principes de base de l'innovation sociale spécifiquement dans le domaine des services est de réfléchir et de mettre en œuvre le service avec l'utilisateur, soit le proche aidant dans ce cas-ci, et non seulement le faire pour eux :

Parce que, dans ce processus-là, pour les proches aidants qui se sont impliqués beaucoup, ça a eu des répercussions personnelles très importantes aussi de reconnaissance par rapport à leur " statut » de proche aidant. Donc, il y a tout ça aussi, je pense, qui est important de travailler. Ce n'est pas juste de recevoir, mais d'avoir une notion d'empowerment avec eux... De les mettre dans une situation où, oui, il y a une reconnaissance. Les gens ne se reconnaissent pas eux-mêmes, souvent. C'est un facteur qui n'est pas nécessairement aidant pour nous. (Entretien 04, intervenante, organisme communautaire)

L'approche ascendante demande beaucoup de temps et de ressources humaines et financières. 
Dans le contexte presque structurel de compressions et de contraintes budgétaires, les intervenants sont souvent obligés de renoncer à ce type d'approche participative ou ne peuvent pas l'appliquer pleinement. La mobilisation citoyenne requiert du temps et des ressources. Les nouvelles initiatives prennent souvent la forme de projets spécifiques et sont financées à travers des appels à projets. Cela permet de développer différentes initiatives dans la région, mais peut également constituer une limite à long terme. Comme nous le soulignons dans notre cadre théorique, il est important que les interventions sociales sur le bien-être local soient organisées dans un programme cohérent. Le fait d'avoir plusieurs petits projets locaux financés tous les deux ou trois ans peut fragmenter les interventions sociales et affaiblir la capacité d'innovation sociale à long terme des organismes locaux.

D'après nos résultats empiriques, la plupart des groupes communautaires ou institutions locales qui ont répondu à des appels à projets pour la mise en place de services pour les proches aidants avaient déjà des activités et préoccupations centrales bien établies; soutenir les proches aidants s'ajoutait donc comme mandat supplémentaire. Dans certains cas, l'intégration de ce mandat était cohérente et bien structurée; dans d'autres cas, le nouveau projet était plutôt une stratégie pour obtenir des fonds additionnels et pour assurer la viabilité financière de l'organisme. Dans ces cas-ci, le nouveau projet risquait plutôt de devenir une surcharge pour les intervenants et l'organisme.

Par ailleurs, même si le processus de mobilisation a permis de mieux cibler et de définir la population aidante, ainsi que de souligner la spécificité et les défis liés à la conciliation travail-famille-soins, les préoccupations spécifiques des aidants travailleurs n'ont que rarement été prises en compte. La plupart des initiatives ne sont pas encore adaptées aux besoins spécifiques de la population aidante qui est active sur le marché du travail. Cette adaptation nécessiterait plus de temps, de ressources, une diversité d'expertises et un partenariat renforcé avec les employeurs, ce qui n'est pas toujours possible dans le contexte actuel.

Cette situation est courante, car les innovations sociales ont besoin de temps pour s'organiser et pour se développer. Tout type d'innovation doit passer par plusieurs étapes d'expérimentation avant d'aboutir à des résultats définitifs et bien établis. Une initiative d'innovation sociale doit être socialisée et partagée à l'aide de différentes stratégies de mobilisation. Une fois qu'une initiative a prouvé son efficacité, son potentiel d'innovation sociale devrait permettre un changement à plus long terme du système de référence et d'action.

De cette façon, l'objectif final d'une innovation sociale est une transformation sociale. Cela ne signifie pas que chaque innovation sociale pourrait ou devrait devenir un service institutionnalisé. Dans l'étude de cas étudiée, le changement dans l'offre de services formels s'est également avéré un obstacle à l'innovation et à la transformation sociale pour améliorer la vie et les conditions des proches aidants.

Lorsqu'une initiative devient un service institutionnalisé et formel, la dimension temporelle peut changer. Les services formels répondent aux besoins sociaux à court terme en suivant souvent un programme politique et les délais du soutien financier. De plus, dans une perspective de changement d'offre de services, les professionnels ont été amenés à gérer des activités administratives, bureaucratiques et financières afin d'assurer la continuité et la viabilité du service. En fin de compte, les relations d'aide et de soutien transforment également l'orientation client-utilisateur.

\section{Conclusion}

Le présent article souligne l'importance de la mobilisation à plusieurs paliers dans la structuration des services aux proches aidants. Le contexte politique a évidemment un rôle fondamental dans la mise en place et dans la pérennité de l'offre de services. Les réformes sociales, compressions budgétaires et d'autres initiatives, comme celles de 2015, peuvent avoir un fort impact (Chiasson et Fortin, 2016; Alberio, 2015; Alberio et Mbaye, 2015). Les ressources économiques ne sont pas seulement nécessaires pour concrètement mettre en place de nouveaux services ou initiatives; elles sont également nécessaires pour le soutien et le maintien de la mobilisation et de la participation citoyenne à différents paliers (Alberio, 2015).

Selon les participants de cette étude, la pérennité de la mobilisation et de la participation citoyenne devenait de plus en plus critique au cours de l'avancement des événements de 2015, notamment à 
cause d'un contexte caractérisé par des ressources financières incertaines, ainsi qu'une incertitude face au cadre institutionnel plus général, avec le démantèlement de l'ancien cadre sociopolitique de concertation locale et régionale, sans qu'il soit remplacé par une nouvelle structure de gouvernance claire.

En ce qui concerne plus spécifiquement les proches aidants, nous soulignons le travail du gouvernement actuel en faveur d'une politique pour le soutien des proches aidants. L'ajout du terme proches aidants au cabinet de la ministre responsable des Ainés et des Proches aidants est un fait symbolique important, bien qu'il soit encore impossible de pouvoir se prononcer sur cette politique en structuration. Cependant, se doter d'une stratégie nationale et de pratiques institutionnalisées pourrait en partie répondre au besoin que nous avons identifié de dépasser la fragmentation territoriale des initiatives et outils.

Un élément qu'il faudrait inverser - et pas seulement dans le cas des proches aidants - est la tendance générale dans l'action publique à négliger des actions à long terme en se concentrant plutôt sur une courte période. Comme conséquence, comme le montre notre analyse, les organismes choisissent aussi d'effectuer des interventions plus bureaucratiques et standardisées, consacrant ainsi beaucoup de temps aux activités de collecte de fonds et à la rédaction de projets pour demander de nouveaux fonds. Cette approche limite non seulement le niveau de mobilisation et de participation citoyenne - indispensables au développement de toute initiative d'innovation sociale -, mais devient également un obstacle à la créativité des professionnels et à leur capacité de planifier et de mettre en œuvre un programme social cohérent et efficace.

En conclusion, nous estimons également que le contexte sociopolitique actuel, avec une propension à la réduction des dépenses - devenue une tendance structurelle, et non simplement une conjoncture dépendant des changements gouvernementaux -, est de plus en plus un obstacle au développement et à la pérennité d'une programmation sociale cohérente capable de bien répondre aux besoins des aidants, en particulier de ceux devant concilier travail-famillesoins.

\section{REMERCIEMENTS}

Nous remercions l' Appui Bas-Saint-Laurent et en particulier Mme Chouinard, directrice générale qui nous a appuyé tout au long de la recherche. Cette recherche est initialement née d'une collaboration avec D.-G. Tremblay (TELUQ) ayant travaillé sur la région de Montréal. Un remerciement important va également aux étudiants et auxiliaires de recherche impliqués dans le projet: Emanuele Lucia, Luce Thériault et Clément Devielhe.

\section{NOTES}

1 L'article suivant est une version adaptée, abrégée et modifiée dans plusieurs parties de Alberio, M. (2018). Supporting carers in a remote region of Quebec, Canada: How much space for social innovation? International Journal of Care and Caring, 2(2), 197-214. https://doi.org/10.1332/239788218X15269112664973

2 Ce modèle centralisé est également reproduit à différents paliers territoriaux, tels que les gouvernements provinciaux au Canada, en particulier au Québec, une province qui contrôle la plupart de ses politiques sociales.

3 La façon dont les responsabilités sont partagées entre différents paliers est centrale, mais aussi conflictuelle, comme nous le verrons dans le cas du Québec. Cela peut concerner les relations entre le gouvernement provincial et ses paliers sous-territoriaux (municipalités et municipalités régionales de comté [MRC]), ainsi qu’avec le secteur communautaire ou associatif.

4 «En 1997, le gouvernement du Québec adopte sa politique de soutien au développement local et régional qui mettait en place une nouvelle structure locale, les centres locaux de développement, où les organismes communautaires étaient invités à se concerter avec les différents partenaires (municipalités, syndicats, institutions, employeurs, etc.) afin de favoriser le développement local, y compris l'économie sociale et solidaire. De plus, cette politique consacrait la participation des organismes communautaires, dans chacune des régions administratives du Québec, aux Conseils régionaux de développement devant permettre à l'État de "consolider le partenariat gouvernement-régions", et ce, dans tous les "secteurs de l'activité gouvernementale susceptibles d'être réorganisés en concertation avec les milieux locaux et régionaux”. »(Québec, 1997, cité dans Bourque, 2009, p. 2)

5 Devenu en 2015 le Centre intégré de santé et de services sociaux (CISSS) du Bas-Saint-Laurent.

6 Les Conférences régionales des élus ont évolué à partir des Conseils régionaux de développement. Elles ont été abolies en mars 2016. 


\section{RÉFÉRENCES}

Alberio, M. (2014). Les travailleurs à faible revenu au Québec : concepts, mesures et aperçu. Dans D.-G. Tremblay et M. Alberio (dir.), Travail et société : une introduction à la sociologie du travail (p. 485-507). Québec, QC : Presses de l'Université du Québec.

Alberio, M. (2015). Les initiatives locales et les défis des acteurs du milieu face aux coupes et aux changements actuels. Organisations et Territoires, 24(3), 53-61. https://doi.org/10.1522/ revueot.v24i3.90

Alberio, M. et Mbaye, O. (2015). Genesi dello sviluppo sociale e territoriale in Québec : un percorso tra continuità e cambiamento. Economia e società regionale, 3(3), 82-98. https://doi.org/10.3280/ES2015-003006

Alberio, M. et Tremblay, D.-G. (2014). Les entreprises d'insertion dans la sortie de crise : entre mission sociale, activité économique et relation avec les pouvoirs publics. Télescope, 20(1), 128-149. Repéré à www.telescope.enap.ca/Telescope/docs/Index/Vol_20_no_1/ Telv20_no1_Tremblay.pdf

Bourque, D. (2009). Transversalité et concertation : entre facteur de développement ou d'enfermement des initiatives locales. Les Politiques Sociales, 1-2, 18-30. Repéré à http://w4.uqo.ca/crcoc/Fichiers/articles/Art_politiques_sociales.pdf

Commission canadienne des droits de la personne (CCDP). (2014). Guide sur la conciliation des responsabilités professionnelles et des obligations familiales des proches aidants : stratégies de collaboration pour un milieu de travail compréhensif et performant. Ottawa, ON : CCDP. Repéré à www.chrc-ccdp.gc.ca/fra/content/guide-sur-la-conciliation-des-responsabilites-professionnelles-et-des-obligations-familiales

Chen, J. et Wilkins, R. (1998). Les besoins des personnes âgées en matière d'aide personnelle. Rapports sur la santé, 10(1) (Statistique Canada, no 82-003 au catalogue), 39-50. Repéré à www150.statcan.gc.ca/n1/fr/pub/82-003-x/1998001/article/3876fra.pdf?st=ZR3hoBXq

Chiasson, G. et Fortin, M.-J. (2016, 14 novembre). La connaissance EN et SUR la périphérie : le Centre de recherche sur le développement territorial [billet de blogue]. Acfas Magazine.

Repéré à www.acfas.ca/publications/decouvrir/2016/11/connaissance-peripherie-centre-recherche-developpement-territorial

Courlet, B. et Pecqueur, C. (2013). L'économie territoriale. Grenoble, France : Presses de l’Université de Grenoble.

Cranswick, K. et Dosman, D. (2008). Soins aux aînés : le point sur nos connaissances actuelles. Tendances sociales canadiennes (Statistique Canada, no 11-008 au catalogue), 50-59. Repéré à www150.statcan.gc.ca/n1/fr/pub/11-008-x/2008002/article/10689fra.pd? st $=$ pLjgUAEf

Ducharme, F. (2006). Famille et soins aux personnes âgées : enjeux, défis et stratégies. Montréal, QC : Beauchemin.

Duxbury, L., Higgins, C. et Schroeder, B. (2009). Balancing paid work and caregiving responsibilities: A closer look at family caregivers in Canada. Ottawa, ON: Human Resources and Skills Development Canada. Repéré à www.cprn.org/documents/51061_ EN.pdf

Fast, J., Lero, D., Duncan, K., Dunlop, C., Eales, J., Keating, N. et Yoshino, S. (2011). Employment consequences of family/friend caregiving in Canada. Edmonton, AB: University of Alberta, Research on Aging, Policies and Practice (RAPP).

Gagnon, E., Guberman, N., Côté, D., Gilbert, C., Thivierge, N. et Tremblay, M. (2001). Les impacts du virage ambulatoire : responsabilités et encadrement dans la dispensation des soins à domicile. Ottawa, ON : Fondation canadienne de la recherche sur les services de santé. Repéré à www.cfhi-fcass.ca/migrated/pdf/researchreports/ogc/gagnon_e.pdf

Gagnon, L. et Savoie, A. (2008). Préparons l'avenir avec nos aînés : rapport de la consultation publique sur les conditions de vie des aînés. Québec, QC : Gouvernement du Québec, Secrétariat aux aînés. Repéré à https://publications.msss.gouv.qc.ca/msss/fichiers/ainee/ F-5149-MSSS.pdf

Institut de la statistique du Québec (ISQ). (2013). Portrait des personnes proches aidantes âgées de 45 ans et plus. Québec, QC : ISQ. Repéré à www.stat.gouv.qc.ca/statistiques/population-demographie/bulletins/coupdoeil-no27.pdf

Institut de la statistique du Québec (ISQ). (2015). Le bilan démographique du Québec. Québec, QC : ISQ. Repéré à www.stat.gouv.qc.ca/statistiques/population-demographie/bilan2015.pdf

Kazepov, Y. (dir.) (2010). Rescaling social policies: Towards multilevel governance in Europe. Farnham, Royaume-Uni : Ashgate Editor.

Klein, J.-L, Fontan, J.-M., Harrisson, D. et Lévesque, B. (2014). L’innovation sociale au Québec : un système d’innovation fondé sur la concertation. Dans J.-L. Klein, J.-L. Laville et F. Moulaert (dir.), L'innovation sociale (p. 193-246). Toulouse, France : Érès. https://doi.org/10.3917/eres.moula.2014.01.0193

Kotsadam, A. (2011). Does informal eldercare impede women's employment? The case of European welfare states. Feminist Economics, 17(2), 121-144. https://doi.org/10.1080/13545701.2010.543384 
Lavoie, J.-P. et Guberman, N. (2009). Le partenariat professionnel-famille dans les soins aux personnes âgées : un enjeu de reconnaissance. Lien social et Politiques, 62, 137-148. https://doi.org/10.7202/039320ar

Lévesque, B. et Mendell, M. (2014). L'économie sociale au Québec : éléments théoriques et empiriques pour le débat et la recherche. Dans B. Lévesque, J.-M. Fontan et J.-L. Klein (dir.), L'innovation sociale : les marches d'une construction théorique et pratique (p. 171-189). Québec, QC : Presses de l'Université du Québec.

Moralli, M., Alberio, M. et Klein, J.-L. (2017). L'innovazione sociale tra sviluppo territoriale e trasformazione sociale. Sociologia urbana e rurale, 113, 30-46. https://doi.org/10.3280/SUR2017-113003

Pavolini, E. (2003). Le nuove politiche sociali : i sistemi di welfare fra istiturioni e società civile. Bologne, Italie : Il Mulino.

Québec. (2009). Loi instituant le fonds de soutien aux proches aidants : RLRQ chap. F-3.2.1.1. [Québec], Éditeur officiel du Québec.

Ranci, C. (2005). Le sfide del welfare locale : problemi di coesione sociale e nuovi stili di governance. La Rivista delle Politiche Sociali, 1(2), $9-26$.

Sinha, M. (2013). Portrait des aidants familiaux 2012 (no 89-652-X). Ottawa, ON : Statistique Canada. Repéré à www150.statcan.gc.ca/n1/fr/pub/89-652-x/89-652-x2013001-fra.pdf?st=fbnN6LrO

Tremblay, D.-G. (2016). Temps de travail, charge de travail et articulation travail-famille : enjeux pour les milieux syndicaux. Revne de l'IRES, 85-86, 145-169. https://doi.org/10.3917/rdli.085.0145

Tremblay, D.-G. et Larivière, M. (2013). La conciliation emploi-famille-soins : quel soutien des entreprises pour les proches aidants? Montréal, QC : Alliance de recherche université-communauté sur la gestion des âges et des temps sociaux (ARUC-GATS). 